\title{
Themenheft: Grundwasser-Oberflächenwasser-Interaktionen
}

\author{
Jan H. Fleckenstein - Christian Schmidt \\ Eingang des Beitrages: 6.7.2009/Online veröffentlicht: 22.7.2009 \\ (C) Springer-Verlag 2009
}

Grundwasser (GW) und Oberflächenwasser (OW) sind Teile eines hydrologischen Kontinuums. Traditionell wurden beide Systeme jedoch häufig getrennt betrachtet und dementsprechend kaum integral und interdisziplinär untersucht. Anfängliche Beschreibungen gekoppelter GW-OW-Systeme waren oft stark vereinfacht. In den letzen 10 bis 15 Jahren jedoch hat das wissenschaftliche Interesse an den Prozessen, die die Interaktion zwischen GW und OW steuern, stark zugenommen. Zum einen die fundamentale Erkenntnis über die sehr spezifischen hydraulischen und biogeochemischen Bedingungen in der Übergangszone zwischen GW und OW (siehe dazu z. B. Special Issue in Hydrological Processes 23 (15), 2009) und deren Bedeutung für die Gewässerökologie, die Wasserqualität und die Wasserwirtschaft sowie zum anderen die Entwicklung neuer Methoden zur Quantifizierung (siehe z.B. Schmidt et al., HESS 10, 849-859, 2006; Vogt et al., diese Ausgabe) und Simulation (siehe Delfs et al., diese Ausgabe) der Austauschprozesse, haben $\mathrm{zu}$ neuen Forschungsansätzen und Aktivitäten

Ph.D. J.H. Fleckenstein $(\bowtie)$

Lehrstuhl für Hydrologie,

Universität Bayreuth,

Universitätsstraße 30, 95440 Bayreuth,

Deutschland

E-Mail: jan.fleckenstein@uni-bayreuth.de

Dr. C. Schmidt

Department Hydrogeologie,

Helmholtz-Zentrum für Umweltforschung

$\mathrm{GmbH}$ - UFZ,

Permoserstraße 15, 04318 Leipzig,

Deutschland

E-Mail: christian.schmidt@ufz.de auf diesem Gebiet geführt. Wichtige Fragen z.B. zu den räumlichen und zeitlichen Mustern des Austausches auf verschiedenen Skalen sowie zum Wechselspiel zwischen Hydraulik und Wasserqualität stellen dabei neue Anforderungen an Feldmethoden und Simulationswerkzeuge. Auch aus Sicht der Praxis ist das Thema von großer Relevanz. Die EU-Wasserrahmenrichtlinie schreibt explizit eine integrale Betrachtung von Grund- und Oberflächenwasserressourcen vor. Das Trinkwasser einiger großer Ballungsräume (z.B. Berlin) wird zu großen Anteilen aus Uferfiltrat gewonnen (siehe Massmann et al., diese Ausgabe). Die Wasserqualität von Restseen in Bergbaufolgelandschaften wird entscheidend durch den Grundwasserzustrom gesteuert (siehe Fleckenstein et al., diese Ausgabe). Das vorliegende Themenheft trägt der zunehmenden Bedeutung und dem gestiegenen Interesse an diesen Themen Rechnung. Natürlich kann ein so weites Themenfeld wie GW-OWInteraktionen dabei nicht allumfassend dargestellt werden. Vielmehr soll ein Überblick über aktuelle Forschungsarbeiten im deutschsprachigen Raum gegeben werden, die innerhalb dieses Themenfeldes von großer Bedeutung sind.

Die Fachbeiträge von Massmann et al. (diese Ausgabe) und Vogt et al. (diese Ausgabe) befassen sich mit dem für die Trinkwassergewinnung bedeutsamen Prozess der Uferfiltration. Massmann et al. (diese Ausgabe) untersuchten die hydraulischen und hydrochemischen Prozesse bei der Uferfiltration an zwei Standorten in der Region Berlin-
Brandenburg. Der Beitrag verdeutlicht die enge Kopplung von Hydrodynamik und Hydrochemie, die zur Ausbildung sehr standortspezifischer Muster in den Verweilzeiten, Redox-Bedingungen und letztendlich der Beschaffenheit des Uferfiltrats führen. Die besondere Bedeutung der Gewässersohle wird dabei hervorgehoben. Im Beitrag von Vogt et al. (diese Ausgabe) werden verschiedene Methoden der Zeitreihenanalyse vorgestellt, die eine Bestimmung der Verweilzeiten und Mischungsverhältnisse zwischen einem infiltrierenden Fluss- und dem angrenzenden Grundwasser anhand von Zeitreihen der elektrischen Leitfähigkeit und Temperatur ermöglichen. Die Anwendbarkeit der Methodik wird an einem voralpinen Fluss in der Schweiz demonstriert. Für Flussabschnitte mit guter Anbindung an das Grundwasser stellt die Methode eine attraktive Alternative zu aufwändigen Markierungsversuchen mit künstlichen Markierungsstoffen dar.

Die Beiträge von Nützmann und Lewandowski (diese Ausgabe) und Fleckenstein et al. (diese Ausgabe) befassen sich mit den räumlichen Mustern und der zeitlichen Dynamik des GW-OW-Austausches. Im Beitrag von Nützmann und Lewandowski (diese Ausgabe) wird die zeitliche Dynamik der GW-OW-Interaktionen an der Spree und einem Altarm der Spree bei Berlin anhand von Wasserstandszeitreihen und einem numerischen Strömungsmodell untersucht. Die kurzeitigen Wasserspiegelschwankungen in der Spree bewirken eine temporäre Umkehr der ansonsten vorherrschenden exfiltrierenden Bedingungen. Im Gegensatz zur 
Spree bewirken die mächtigen Mudden im Altarm, ähnlich wie in dem Beitrag von Massmann et al. (diese Ausgabe) für die Havel beschrieben, dass Abschnitte des Oberflächenwassersystems von Grundwasser unterströmt werden. Fleckenstein et al. (diese Ausgabe) untersuchen die räumlichen und zeitlichen Muster des Austausches zwischen einem sauren Restsee und dem GW. Die bestimmten Austauschraten weisen sowohl kleinräumig $(<5 \mathrm{~m})$ als auch auf den gesamten See bezogen eine hohe Variabilität auf. Veränderungen des Seewasserspiegels bewirken in Teilbereichen des Sees, die vermutlich an unterschiedliche Grundwasserstockwerke angebunden sind, unterschied- lich stark ausgeprägte Schwankungen der Austauschraten.

Im Beitrag von Delfs et al. (diese Ausgabe) werden Konzepte und Ansätze zur voll integrierten numerischen Simulation gekoppelter GW-OW-Systeme vorgestellt. Anhand von Testsimulationen verschiedener GW-OW-Systeme wird die Anwendbarkeit des Kompartimentkonzepts demonstriert. Der Beitrag verdeutlicht das große Potenzial aber auch die rechentechnische Herausforderung, die die Anwendung derartiger voll-integrierter numerischer Modelle in gekoppelten GW-OW-Systemen darstellt.

Die in diesem Sonderheft enthaltenen Beiträge spiegeln exemplarisch die hohe Komplexität von GW-OW-Inter- aktionen wieder. Neben der zeitlichen Variabilität, vor allem durch die höhere Dynamik des Oberflächenwassers bedingt, bewirken Heterogenitäten auf verschiedensten Raumskalen sehr spezifische räumliche Muster des Austausches. Diese Prozesse auf den relevanten Skalenebenen adäquat zu charakterisieren, quantifizieren und simulieren stellt eine Herausforderung dar. Wir hoffen, dass das vorliegende Sonderheft die wissenschaftliche Diskussion zu diesem Thema im deutschsprachigen Raum anregen wird und damit auch einen Beitrag zur Lösung angewandter Probleme liefern kann. 